Original Research Article

\title{
Microbial Resources and Ecology - Microbial Degradation of Pesticides
}

\author{
Xiaolan Ye,Fei Dong,Xiangyang Lei \\ School of Environment and Planning, Wuxi Agricultural University, Jiangsu, China
}

\begin{abstract}
Pesticides play an important role in preventing insect pests and weeds in crops. However, due to the serious threat of pesticide residues to environmental and human health, this paper reviews the hazards of pesticide residues, the mechanism of microbial degradation of pesticides, the factors that affect the degradation of pesticides and the new application of microbial degradation of pesticides.

KEYWORDS: pesticide pollution; microorganism; degradation mechanism; biodegradation; new technology
\end{abstract}

\section{Pesticide residues and their hazards}

The first 'green revolution' in the late 1960s made a significant contribution to human food production. [1, 2] The application of pesticides ensured the grain production. Pesticides as the main agricultural production to rely on, in agriculture has a wide range of applications. At present, the application method of pesticide in our country is still based on the spraying of liquid and the spraying of powder. The research shows that [1]: Only $1 \%$ to $2 \%$ of the drug is used to control the target body, $10 \%$ to $20 \%$ On the body of the crop, 80 to 90 percent of the pesticide is mainly scattered in the surrounding environment of the crop, such as farmland, soil or floating in the atmosphere, and adsorbed with dust to form aerosols. After the probation of pesticides after a period of degradation, metabolism and other effects, its content will be reduced to a certain level, usually, we residual in the environment and the body of trace pesticides called residual pesticides, which include pesticide residues and the amount of the degradant that has a higher or comparable toxicity than the parent. Pesticide pollution is one of the most influential organic pollution in China, which directly threatens the living environment and health of human beings. It is also one of the main reasons for the trade barriers of agricultural and sideline products in China.

Since Taylor and others found DDT efficient insecticidal ability, different types of pesticides have been developed and applied. Only insecticides currently have four categories: organochlorines, organophosphorus, pyrethroids and carbamates. Organochlorine pesticides are high-residue, highly bio-rich class of pesticides that are currently banned from production, but have been used extensively for many years, so there are still residues in the environment, environmental and human threats Very big. Organophosphorus, pyrethroids and carbamate pesticides, although the residual period is short, but there is still a certain period of time, coupled with the widespread use of the soil, the atmosphere and water caused by varying degrees of pollution.

Ge Feng [4] the pesticide hazards generally attributed to four aspects: (1) beneficial biological was killed, the control effect weakened. (2) Increased pest resistance, increased cost of prevention and treatment, increased environmental pollution. (3) Pesticide residues in agricultural and sideline products increased, endangering human health. (4) Pollution of the soil, the atmosphere and water, seriously damaged our living environment. According to the results of the first national pollution survey, agricultural pollution has become China's largest source of pollution, and pesticide pollution has become an important factor in agricultural pollution [5]. As the fruits, vegetables and other pesticide residues exceeded the prevalence of pesticide poisoning incidents have occurred, seriously affecting human health. Pesticide residues have caused widespread concern. Looking for effective ways of pesticide degradation, to solve the problem of pesticide residues is an important issue that scientists need to face and urgently need to solve.

Copyright (C) 2017 -. This is an Open Access article distributed under the terms of the Creative Commons Attribution-NonCommercial 4.0 International License (http://creativecommons.org/licenses/by-nc/4.0/), permitting all non-commercial use, distribution, and reproduction in any medium, provided the original work is properly cited. 


\section{Influencing factors of pesticide degradation}

Pesticides in the environment are mainly adsorption and degradation of two kinds of where the degradation of pesticides can be divided into biodegradable and non-biodegradable two ways. In the light, heat and chemical factors under the action of degradation of the phenomenon of non-biodegradable; and in plants and animals or micro-organisms in vivo degradation of biodegradation. Biodegradation is mainly microbial degradation in pesticide degradation to occupy a dominant position.

(1) Environmental factors: pesticides into the environment, will be subject to a number of environmental factors, such as: temperature, humidity, $\mathrm{pH}$, water content, organic matter content, viscosity and climate. Generally, in the case of high temperature and humid, organic matter rich, $\mathrm{pH}$ alkaline, pesticide is easy to be degraded low. Thomas and Jennifer were used to study the degradation of the soil in the soil, and the degradation efficiency of the pesticide in the soil was improved when the soil was added with compost, stem and sawdust to improve the organic matter content.

(2) The factors of pesticides: pesticide molecular structure, the use of pesticide concentration and pesticide drug history also affect the degradation performance of pesticides. Pesticides because of its molecular structure and physical and chemical properties of different biodegradability sensitivity is very different. For example, 2,4-D and 2,4-T, due to the addition of 1 chlorine atom at the 5 th $\mathrm{C}$ atom, the time required for degradation is increased from $14 \mathrm{~d}$ to $200 \mathrm{~d}, 2,4,5-\mathrm{T}$ It is quite difficult to degrade by microorganisms.

(3) The impact of micro-organisms: the main way of degradation of pesticides is carried out under the action of microorganisms, so the degradation of pesticides have a significant impact on pesticides. The variety of microbes, the number of large, is conducive to the degradation of pesticides. Wang Qian et al., isolated from the activated sludge and a strain of Pseudomonas aeruginosa, mixed bacteria than the single bacteria degradation rate, the removal rate of methamidophos up to $95.15 \%$ to $97.14 \%$.

\section{Microbial degradation}

Biodegradation studies began in the 1940s, and it was initially thought that biodegradation refers to the destruction or mineralization of aerobic microbes in the soil, water and wastewater biological treatment systems for natural and synthetic organic matter. With the deepening of the degradation of organic pollutants, the connotation of biodegradation is deepening and expanding. Since the role of microorganisms in the various biodegradation is greatest, it is generally mentioned that biodegradation mainly refers to microbial degradation [6]. On the current level of technology, chemical pesticides in the future for a long time or irreplaceable products. Therefore, the solution of pesticide residues in the environment has become a hot topic in the world. Among them, the microbial repair is universally recognized as a safe and effective method of remediation of soil pollution [7].

\subsection{The mechanism of microbial degradation of pesticides}

Microorganisms on the role of pesticides can be divided into two categories [8], a class of microorganisms directly on the role of pesticides, pesticide degradation by enzymatic reactions, often said that pesticide microbial degradation belong to this category; one is through microbial activity Changed the chemical and physical environment and indirectly applied to pesticides. Common modes of action are mineralization, co-metabolism, bioconcentration or cumulative effects and microbial effects on pesticides. Microbial degradation of pesticides through enzymatic reactions are mainly oxidized, dehydrogenation, reduction, hydrolysis, synthesis and other types of reactions.

When the degradation of microbial pesticides is caused by its intracellular enzymes [9], the whole degradation process through three steps: (1) the adsorption of pesticides on the surface of microbial cells, the process is a dynamic balance, but also lead to the early degradation The critical phase of the lag phase. (2) Pesticides penetrate the cell membrane into the membrane, the bacteria in a certain amount of pesticide on the cell membrane permeability determines its penetration of the cell membrane, pesticide penetration of the cell membrane is the degradation rate limit step. This penetration rate of pesticides is closely related to the molecular structure parameters of pesticides (mainly lipophilic parameters and steric hindrance parameters). (3) Pesticides in the cell membrane through the combination with the degrading enzyme enzymatic reaction, which is a rapid process.

\subsubsection{Mineralization}

There are many chemical pesticides are natural compounds analogues, can be used as a source of microbial nutrients by microbial decomposition, the formation of inorganic, carbon dioxide and water. Mineralization is the best way to degrade. This is because the pesticide is completely degraded into non-toxic inorganic matter. Shi Lili et al [10] studied the degradation of methyl parathion and its degradation mechanism in Pseudomonas vaginalis DLL-1. It was pointed out that DLL-1 bacteria could completely degrade methyl parathion into NO2- and NO3-. 


\subsubsection{Co-metabolism}

Some of the synthetic compounds cannot be degraded by microorganisms, but if there is another carbon matrix and energy for the presence of auxiliary matrix, they can be partially degraded, this effect is called co-metabolism [11]. The degradation of the monomethylamine product of Pseudomonas dactylus DR-8 was 2,4-dimethylaniline and $\mathrm{NH} 3$, and the DR-8 strain could not grow with monomethylamine as carbon source and energy, Other organic nutrient matrix as a carbon source under the conditions of degradation of methamidine, and the degradation products are not fully mineralized, belonging to the common metabolic type [12]. The main principle of using microbial remediation of environmental pesticide pollution is the use of organic pesticides as a carbon source, nitrogen source, the complex pesticide compounds into simple compounds, or completely decomposed into $\mathrm{CO} 2, \mathrm{H} 2 \mathrm{O}, \mathrm{NH} 3$, thereby reducing pesticide residues in the environment And toxicity [13]. Lin Gan and other cypermethrin as the target pollutants, through enrichment and culture of aerobic treatment tank sludge, obtained a better degradation of the pollutants of the mixed culture of microorganisms, with the microbial degradation of bacteria, the cypermethrin biodegradation The characteristics of the experimental study. The results showed that the mixed culture could improve the degradation rate of cypermethrin by using cypermethrin as the only carbon source, nitrogen source and energy source, and the appropriate amount of carbon source could increase the degradation rate of cypermethrin [14].

\subsubsection{Other microbial degradation pathways}

\subsubsection{Hydrolysis}

Under the action of microorganisms, the ester bond and the dialkylamine bond hydrolyze, so that the pesticide detoxification, such as malathion, propanil and other degradation.

\subsubsection{Dehalogenation}

Fecal hydrocarbon pesticides, in the role of the enzyme, the substituents on the halogen by the $\mathrm{H}$ atom or carboxyl, to replace the loss of toxicity, such as DDT degradation into DDE is such a reaction.

\subsubsection{Oxidation}

Microorganisms through the synthesis of oxidase, the molecular oxygen into the organic molecules, especially organic molecules with aromatic ring, insert a hydroxyl or form an epoxide, such as carbendazim and 2,4- D degradation.

\subsubsection{Nitro reduction}

$\mathrm{N} 2 \mathrm{O}$ in the pesticide to $\mathrm{NH} 2$, such as 2,4-dinitrophenol, the degradation products for the 2 - amino - 4 - nitrophenol and 4 - amino - 2 - nitrophenol; parathion to amino - phosphorus.

\subsubsection{Methylation}

Add toxic phenols to methyl groups to passivate them, such as pentachlorophenol, tetrachlorophenol and other degradation.

\subsubsection{Demethylation}

Containing methyl or other hydrocarbon groups, connected to $\mathrm{N}, \mathrm{O}$ and $\mathrm{S}$, to remove these groups into non-toxic substances, such as dexamethasone degradation of the removal of two N-methyl.

\subsection{Commonly used pesticide degradation of microorganisms}

Microorganisms play the most role in a variety of biodegradable, so far, a variety of biodegradable pesticides have been isolated, including bacteria, fungi, actinomycetes and algae [15]. Bacteria have a variety of biochemical capacity, easy to mutagenesis, in the biological restoration of the main position, of which the most active Pseudomonas strains, a variety of pesticides $\backslash \backslash$ fungicides and herbicides [16] play a highly effective degradation The At present, the study of bacteria is more extensive. Table 1 is the degradation of common pesticides for biology [17]. 
Table 1. Common pesticide degradation microbes

\begin{tabular}{|l|l|}
\hline \multicolumn{1}{|c|}{ Pesticide } & \multicolumn{1}{|c|}{ Degradation of Microorganisms } \\
\hline Methicillin & Aspergillus, Penicillium, Pseudomonas, Bottle Yeast \\
\hline Atorvastatin (AT) & $\begin{array}{l}\text { Aspergillus fumigatus, Aspergillus fumigatus, Rhizopus oryzae, Fusarium moniliforme, Fusarium } \\
\text { graminearum, Fusarium oxysporum, Fusarium graminearum, Penicillium purpureum, Penicillium } \\
\text { cristatum, Penicillium sp, White rot fungi, mycorrhizal fungi, Pseudomonas, Rhodococcus, Nocardia }\end{array}$ \\
\hline Urea 3 & Fungi \\
\hline The enemy is killed & Alcaligenes \\
\hline 2,4-D & $\begin{array}{l}\text { Pseudomonas, Lactobacillus, Arthrobacter, Corynebacterium, Flavobacterium, Rhodobacter sphaeroides, } \\
\text { Streptomyces, Aspergillus, Nocardia }\end{array}$ \\
\hline DDT & $\begin{array}{l}\text { Acinetobacter, Bacillus, Bacillus, Clostridium, Escherichia, Pseudomonas, Proteus, Streptococcus, } \\
\text { Lactobacillus, Xanthomonas, Erwinia, Pasteurella, Agrobacterium tumefaciens, Aerobacter aerogenes, } \\
\text { Fusarium mold, Nocardia and Trichoderma viride }\end{array}$ \\
\hline Fumo six or six & White rot fungi, Clostridium, Escherichia coli, Escherichia coli, Clostridium \\
\hline Parathion & Escherichia coli, Bacillus \\
\hline Heptachlor & Bacillus subtilis, Fusarium moniliforme, Micromonospora, Nocardia, Aspergillus, Rhizopus, Streptococcus \\
\hline Trichoderma spp & Pseudomonas aeruginosa \\
\hline Dieldrin & Bacillus, Pseudomonas \\
\hline Aldrin & Fusarium mold, Penicillium \\
\hline Dimethoatet & Pseudomonas \\
\hline $2,4,5-T$ & Lactobacillus \\
\hline
\end{tabular}

\subsection{Factors that affect the degradation of pesticides by microorganisms}

\subsubsection{The impact of microbes themselves}

The microbial species, metabolic activity, environmental adaptability and so on have a direct impact on the degradation of pesticides, and microbial spatial distribution, population density and interaction with other microorganisms have determined the degradation efficiency of microorganisms. A large number of experiments have shown that different microbial species or the same species of different strains of the same organic substrate reactions are very different [18]. Microorganisms develop the ability to degrade through a certain adaptation process, the introduction of new compounds, or the generation of corresponding enzymes by gene mutations under the ability of strong adaptation and domestication [19]. The functional characteristics and changes of microbial degradation are also important factors influencing the degradation ability and mechanism. Microbes because of their own habits [20] on the degradation of different pesticides have different results, which is the researchers generally concerned about the problem, with the deep understanding of micro-organisms, pesticide degradation research will be developed by leaps and bounds.

\subsubsection{Effects of Pesticide Structures}

The molecular weight of the pesticide compounds, the structure of the space, the type and number of substituents affect the difficulty of degradation of microorganisms [15]. Under normal circumstances, the polymer compounds than the low molecular weight compounds difficult to degrade, polymers, complexes more resistant to biodegradation [21]; simple structure than the complex structure of the complex degradation [24]. Chen Yali et al. [22] found that compounds with $-\mathrm{OH}$ or $-\mathrm{NH} 2$ on the benzene ring were more susceptible to degradation by Pseudomonas sp. WBC-3, which was usually prehydroxylated and re-opened Principle consistent. Potter et al. [22] studied the degradation of PAHs under small compost conditions. It was noted that the aromatics of 2-4 rings were more likely to degrade than the 5-6 ring aromatics.

Most of the present environmental pollutants are synthetic biologically heterologous organic substances that do not exist in nature, and tend to exhibit strong resistance to microbial degradation. The reason may be that these compounds are relatively short, Microorganisms have not evolved to degrade the metabolic mechanisms of these compounds. Although some dangerous compounds may be degraded slowly in nature by the synergistic action of naturally occurring microbial populations, this remains a new challenge for the microbial world. The process by which microorganisms are able to degrade the capacity of a compound by changing its own information is slow, and the natural evolutionary process of microbes is clearly unable to meet the requirements compared to the currently widely used synthetic heterologous substances. Therefore, Will cause the imbalance of the entire ecosystem [23]. Therefore, it is very important and urgent to study some methods that enable microbial populations to achieve maximum degradation of the isoforms in a relatively short period of time. 


\subsubsection{Impact of environmental factors}

Pesticide pollution and its degradation of microorganisms in which the environment temperature, humidity, $\mathrm{pH}$, containing $\mathrm{C}$ and $\mathrm{N}$ and other organic matter content, salinity, matrix adsorption, viscosity and ventilation, can affect the degradation of pesticides pollution of pesticides The Most microorganisms have the optimum growth temperature, and the degrading enzyme also has the optimum reaction temperature. The change of temperature can affect the metabolism of microorganisms, the enzyme activity of degrading enzymes and even the physical state of pesticide pollution, thus affecting the degradation rate. In general, $\mathrm{pH}$ acidic, which is conducive to fungal reproduction, $\mathrm{pH}$ alkaline or neutral, conducive to bacterial reproduction. PH alkaline, organic phosphorus, organochlorine pesticides themselves are susceptible to degradation, residual low. In the humid, rich organic matter content, well ventilated environment, conducive to aerobic or facultative anaerobic microbial growth. Mo-hui et al [24] pointed out that the activity of microbial degradation of polycyclic aromatic hydrocarbons in compost is closely related to the concentration and moisture content of oxygen. When the content of oxygen in compost is less than $18 \%$ and the moisture content is more than $75 \%$, compost is from aerobic Conditions into anaerobic conditions, thereby affecting the degradation of PAHs. Kastner et al. [25] argue that the effect of organic substrate content on pesticide contamination degradation in composting is greater than that in composting for the degradation of pesticide contamination in the case of composting with soil contaminated with PAHs. Co-metabolic degradation of pesticide-contaminated microorganisms is more important because microbes do not produce energy when metabolically reducing pesticide contamination, requiring additional carbon and energy sources to replenish energy.

\subsection{Microbial degradation of pesticide technology}

\subsubsection{Application of transgenic technology}

Molecular biology, genetics and other disciplines of rapid development led to the emergence of a variety of biotechnology, coupled with bioinformatics, proteomics, genomics and other new disciplines quickly rise, are people to create 'super pesticide pollution degradation bacteria' provides a good condition. The mechanism of degradation and regulation of degrading enzyme gene was clarified at the DNA level, and more degradation genes were cloned and the highly degraded microorganisms were prepared. The results showed that the degradation mechanism of the biodegradable microorganisms and the pesticide pollution degradation plasmids and pesticide pollution-degrading enzymes were more deeply studied. Of the gene pool, the use of modern genetic engineering to build more efficient 'pesticide pollution degradation engineering bacteria' or 'pesticide pollution degradation enzyme expression system', broaden the degradation spectrum, improve the degradation capacity [26].

\subsubsection{Construction and Application of Multi - strain Complex System}

Previous studies on biodegradation of pesticide contamination have focused on pure culture with a single microbial strain, and it has now been shown that the pure culture of a single strain is not as well as mixed culture. Because a single microorganism does not have the genetic information of all the enzymes required for biodegradation, and their time of acclimation in the refractory compound is not sufficient to evolve the complete metabolic pathway, many pure cultures have found that during biodegradation Toxic intermediate material accumulation, so thorough mineralization usually requires one or more of the nutrient flora (such as fermentation - hydrolysis bacteria, sulfur bacteria, acetic acid producing bacteria and methanogens). A part of the microbial degradation, through several microbial relay and synergistic effect, after a multi-step reaction to the toxic compounds completely mineralized, microbial community action more resistant to biodegradation of toxic substances [27].

\subsubsection{Application of immobilized microbial technology}

Immobilized microbial technology is a new type of biotechnology that emerged in the 1980s by locating free cells or enzymes in a defined spatial region by chemical or physical means to keep them active and reusable, Bioreactor microbial cell concentration and purity, and to maintain high efficiency bacteria, secondary pollution and other characteristics. The use of immobilization technology to fix the degradation of microorganisms and enzymes, and then used to deal with pesticides contaminated soil or water, has good prospects for development.

\section{Microbiological problems in the degradation of pesticides}

Although pesticide residues in microbial degradation research has made great progress, but also has some examples of applications, but most of the research in the laboratory, pesticide degrading bacteria completely out of the laboratory to the practical application of a way to go. Pesticide microbial degradation of the main problems are the following aspects. 


\subsection{Pure culture of single strain}

Previous studies have focused on pure culture of a single strain, obtaining purely cultured strains in the laboratory, and then studying its properties and degradation mechanisms. However, this method is completely inconsistent with the actual situation, the natural state, is a variety of microbial coexistence, through the interaction between the microbial degradation of pesticides [28]. Pesticide residues are often present in complex environments such as soil, agricultural and sideline products and waste. [29] Even if the degradation activity of a strain in the laboratory is large, it may not survive or fail to achieve the desired effect under this complex condition The Therefore, the viability and degradation activity of microorganisms in the actual environment need to be further studied, and it is necessary to determine whether the selected strains belong to the dominant species [30].

\subsection{Effects of environmental conditions on microbial degradation of pesticides}

The external environment has great influence on the growth of microorganisms and the degradation of pesticides, such as the temperature, moisture content, $\mathrm{pH}$ and oxygen content of the environment, and these factors in the natural environment change greatly, which directly affects the degradation of pesticides. How to overcome the impact of the environment in order to give full play to the role of target microorganisms is a major problem to be solved.

\subsection{Effect of Biodegradation of Target Compounds on Degradation}

Whether the concentration of the target compound can cause the growth of the microorganism, and the compound composition of the pesticide contaminated environment is very unstable and fluctuates greatly, which makes it difficult to project the microbial degradation of the pesticide compound.

\subsection{The degree of difficulty of contact of microorganisms with degraded substances}

Pesticide contaminated environment with soil, air, water and fruits and vegetables, for soil and water pollution, microbes are easy to contact with pollutants, and thus play their degradation function. However, for pesticidecontaminated food, it is difficult to use residual microorganisms to degrade residual pesticides because microbes cannot come into contact with residual pesticides present in the object and cannot exert their effects, but only the parts that remain on the surface of the object. This restriction requires people to solve as soon as possible, thereby expanding the scope of application of microbial degradation of pesticides.

\subsection{Adaptability of microorganisms}

Whether the vaccinated microorganisms can adapt to the polluted environment, not only the physical environment mentioned above, but also the relationship between organisms. The microbes that are inoculated into the environment are affected by the inhibitory substances, or are affected by the indigenous microorganisms, including the predator, and are not even antagonistic, which can cause the inoculated microorganisms to become a dominant bacterium and thus lose their pesticide Degradation. Construction of multi-strain complex system, with the advantages of stability and strong anti-pollution, but even the multi-bacteria mixed culture of the complex system also exist can become a dominant group of problems.

\section{Development Trends and Prospects}

Microbial degradation of pesticides has been developed at present. Microbial strains of various degraded pesticides have also been isolated and identified. However, the practical application of bioremediation by microbes is often affected by its low degradation efficiency. In recent years, with the development of genetic engineering and molecular biology research technology, researchers began to shift the focus to the construction of highly efficient engineering bacteria, using gene recombination technology, the expression of highly efficient degradation of pesticide enzyme gene into the carrier, Transformation of engineering bacteria, in order to improve the degradation of specific protein or enzyme expression levels, thereby improving the degradation efficiency, both to overcome the enzyme in the environment can not be stable problems, but also to maintain the high activity of the enzyme. In short, the use of genetic engineering technology so that people can be set up in accordance with the needs of human beings with special function of the degradation of the plasmid, resulting in high degradation efficiency, degradation of a wide range of stable expression of the new strain. With the further development of genetic engineering, the potential of microorganisms in pesticide degradation will be more fully reflected. 


\section{References:}

1. Xi Zhijiang, Li Qiuuo. Pesticide microbial degradation technology research [J]. Journal of Yangtze River University b: Natural Science Edition, 2007, (2): 87-90.

2. Liu Jianli. Using microbial degradation of pesticide pollution [J]. Biology Teaching, 2010, (4).

3. Liu Lijie, Qin Dezhi, Jin Rong et al. Advances in microbial degradation of organic pesticides [J]. Inner Mongolia Forestry Science and Technology, 2013, (4): 34-36.

4. Ge Feng, Li Dianmo, Ding Yanqin. Pest damage and its prevention and treatment of new benefits [C]. // Towards the 21st century Chinese Entomology - China Entomological Society Academic Annual Meeting.

5. Li Yang, Wang Yuling, Li Jingmiao. Study on Soil Pollution and Bioremediation Technology of Organic Pesticides [J]. Journal of China Environmental Management Cadre College, 2009, (3): 64-66.

6. YOU Min-sheng, LIU Xin. Biodegradation and bioremediation of pesticide pollution [J] .Chinese Journal of Ecology, 2004, 23 (1): 73-77.

7. Huang Yali, He Xianke, Zhang Liping, et al. Pesticide contaminated soil microbial remediation research progress [J]. Modern agriculture, 2011, (9).

8. Arisoy M. Biodegradation of chlorinated organic compounds by white-fung fungi [J]. Bull. Environ. Contam. Tellurol, 1998, 60: 875-876.

9. Yang Xiaohong, microbial degradation of pesticide research progress, microbiology, 2003.30 (7): 93 - 96.

10. SHI Li-li, LIN Yu-lock, XU Yi-gang et al. Degradation of methyl parathion pesticide by DLL-1 bacteria and its degradation mechanism [J]. Journal of Ecology and Rural Environment, 2002, (3).

11. Zhu Fuxing, Wang Mo, Li Jianhong. Microbial degradation of pesticides [J]. Microbiology Bulletin, 2004, 31 (5): $120-123$.

12. Xi Beidou, Liu Hongliang. Study on preparation of $\mathrm{TiO} 2$ semiconductor photocatalyst by hydrolysis of tetrabutyl titanate [J] .Acta Scientiae Circumstantiae, 2001, 21 (2): 144-147.

13. Chang Xuexiu, Zhang Hanbo, Yuan Jiali. Environmental pollution microbiology [M]. Beijing: Higher Education Press, 2006: 157-158

14. Chinese Journal of Chemistry and Biotechnology, 2005 (8): 40-42 (in Chinese with English abstract) [J].

15. FAN Rong-gui, LIU Bo, WANG Quan-cheng, et al. Immobilization technology of white rot fungi and its research progress [J] .Guangdong Chemical Industry, 2011, (10): 65-66.

16. Wang Guangli, Chen Honghong, Bi Meng, et al. Preparation of Pseudomonas sp. Strain CTN-3 to polluted soil of chlorothalonil [J]. Chinese Journal of Applied Ecology, 2012, (3).

17. Journal of Liaoning Agricultural Sciences, 2013, (4): 52-55 (in Chinese with English abstract).

18. Analysis of polycyclic aromatic hydrocarbons (PAHs) in municipal sludge and corn stalk compost [J]. Journal of Agricultural Engineering, 2001,17 (5): 73-77.

19. Kong Fanxiang, Yin Dajiang, Yan Guoan. Environmental Biology [D]. Beijing: Higher Education Press, 2000

20. [J]. Journal of Shandong Agricultural University: Natural Science Edition, 2012, (4): 519-524.

21. Kong Fanxiang, Yin Dajiang, Yan Guoan. Environmental Biology [M]. Beijing: Higher Education Press, 2000.211-230.

22. Potter C.L., Glaser J.A., Chang L.W., et al. Degradation of polynuclear armotic hydrocarbons under bench-scale compost conditions [J]. Environmental Science and Technology, 1999, 33 (10): 1717-1725.

23. Chen Jian. Environmental Biotechnology [M]. Beijing: China Light Industry Press, 1999.279 - 319.

24. (Institute of Agricultural Engineering, Zhejiang University, Hangzhou 310027, China); Study on Polycyclic Aromatic Hydrocarbons (PAHs) in Municipal Sludge and Corn Stalk Compost [J]. Journal of Agricultural Engineering, 2001, (5): 73-77.

25. Johnsen A R, Karlson U. PAH Degradation Capacity of Soil Microbial Communities-Does It Depend on PAH Exposure? [J]. Microbial Ecology, 2005, 50 (4): 488-495.

26. Wang Shenghui, Zhang Chen, Yan Yanchun. Advances in microbial degradation of organophosphorus pesticides [J]. Biotechnology, 2006, (3): 95-97.

27. Wang Weidong, Niu Junling, Cui Zongjun. Pesticide microbial degradation [J]. Journal of Heilongjiang August First Land Reclamation University, 2005, (2).

28. ZHENG Jin-lai, LI Jun-wen, CHUO Fu-huan. Progress and Prospect of Pesticide Degradation Microorganisms [J]. Environmental Science Research, 2011, (2): 62-64.

29. Su Shaohui. Microbial degradation of pesticide research status and development trends [J]. Journal of Suzhou Education College, 2014, (4).

30. Liu Jianli. Using microbial degradation of pesticide pollution [J]. Biology Teaching, 2010, (4). 